\title{
Oncoprotein Bcl-2 and microsatellite instability are associated with disease-free survival and treatment response in colorectal cancer
}

\author{
RIYAD BENDARDAF, HANAN LAMLUM, RAIJA RISTAMÄKI, \\ KARI SYRJÄNEN and SEPPO PYRHÖNEN \\ Department of Oncology and Radiotherapy, Turku University \\ Central Hospital, Savitehtaankatu 1, PB 52, FIN-20521, Turku, Finland
}

Received April 16, 2008; Accepted May 20, 2008

DOI: 10.3892/or_00000101

\begin{abstract}
Colorectal cancer (CRC) cell lines displaying microsatellite instability (MSI) are resistant to 5-fluorouracil in vitro, which can be overcome by restoring DNA mismatch repair (MMR) competence. Furthermore, elevated levels of Bcl-2 protein confers cytotoxic drug resistance to tumour cell lines. We examined the expression of Bcl-2 and two MMR proteins (hMLH1 and hMSH2) in advanced CRC patients, to determine their mutual relationship, association to therapeutic response and impact on disease outcome. Tumour samples from 73 CRC patients who were treated in advanced stage with either irinotecan alone or in combination with 5-FU/leucovorin, were analysed for expression of Bcl-2, hMLH1 and hMSH2 using immunohistochemistry. Bcl-2 expression was closely correlated with hMLH1 and hMSH2 expression (negative-weak/moderate-strong) $(\mathrm{p}=0.01)$. Bcl-2/MMR expression was significantly $(\mathrm{p}=0.030$ for whole series; $\mathrm{p}=0.018$ for the 5 -FU-treated cases) related to the response to treatment; tumours with low levels of both Bcl-2 and MMR responded better $(n=18 / 31,58 \%)$ than those with high Bcl-2 and MMR ( $n=3 / 16,18 \%)$. Patients with high Bcl-2/MMR expression had a significantly longer DFS (47 vs. 11 months, $n=26$ ) than those with low Bcl-2/ MMR index $(p=0.005)$. Bcl-2/MMR index was not significantly related to disease-specific survival or survival with metastases. The present data suggest that MSI patients with low Bcl-2/MMR demonstrate a significantly shorter DFS, whereas patients with high expression of the two markers obtain the greatest benefit from 5-FU-based chemotherapy.
\end{abstract}

Correspondence to: Dr R. Bendardaf, Department of Oncology and Radiotherapy, Turku University Hospital, Savitehtaankatu 1, PB 52, FIN-20521, Turku, Finland

E-mail: riyben@utu.fi

Key words: colorectal cancer, 5-fluorouracil, response to therapy, Bcl-2, hMLH1, hMSH2, prognosis

\section{Introduction}

Colorectal cancer (CRC) is the second most frequent cancer in Europe in 2004, responsible for $13 \%(376,400)$ of all incident cancer cases. It is also the second most frequent cause of cancer mortality in Europe, with $11.9 \%(203,700)$ annual deaths (1). In the early stages, CRC is often a curable disease, but the overall prognosis is determined by the extent of local and particularly metastatic tumour spread. However, disease outlook is relatively poor for advanced disease and thus is a significant cause of worldwide cancer-related mortality (1). For locally advanced and metastatic CRC, fluoropyrimidine, 5-fluorouracil (5-FU) has been the standard cytostatic drug for the past 50 years, being currently used as modulated by leucovorin or in combination with irinotecan or oxaliplatin.

Increased expression of the proto-oncogene Bcl-2, a $24 \mathrm{kDa}$ intracellular membrane protein that is able to inhibit programmed cell death without affecting cell proliferation, has been reported in gastrointestinal adenocarcinoma and its precursor lesions (2,3). Bcl-2 has been shown to prolong cell survival by inhibiting apoptosis in several cell types $(4,5)$. Abnormal activation of the $\mathrm{Bcl}-2$ gene appears to be an early event in colorectal tumorigenesis (3).

Microsatellite instability (MSI) is observed at the nucleotide level, frequently resulting in deletions or insertions of a few nucleotides, leading to MSI. The inherent instability of microsatellite loci is primarily due to changes in the number of repeats during DNA replication because of inefficiencies in the DNA mismatch repair (MMR) system. This system normally identifies and repairs errors that may occur during DNA replication. The loss of one of the DNA mismatch repair genes leads to the rapid accumulation of mutations and an increased risk of tumorigenesis, for example colorectal cell lines deficient in MMR show higher accumulation of other mutations and deletions $(6,7)$.

In this study, we examined the expression of oncoprotein Bcl-2 and the expression of two MMR genes, hMLH1 and hMSH2 in locally advanced and metastatic CRC patients, to determine their inter-relationships as well as their impact on patient survival and treatment response. 


\section{Patients and methods}

Patients, treatment and follow-up. A series of 73 patients were diagnosed and treated for stage II, III and IV CRC at the Department of Oncology and Radiotherapy, Turku University Hospital (TUH) and six other hospitals of the same hospital district, between January 1996 and August 2003. The key clinical characteristics of the patients are summarized in Table I.

At the time of diagnosis, 12 patients had stage II, 14 had stage III and 47 had stage IV disease. When metastases or inoperable local recurrence were observed, patient were entered into the chemotherapy protocol. In the protocol, patients received one of two treatment regimes; 20 received irinotecan alone and 53 received a combination of irinotecan, 5-fluorouracil (5-FU) and folinic acid (FA) as first-line treatment for metastatic disease. Irinotecan $\left(350 \mathrm{mg} / \mathrm{m}^{2}\right)$ was administered as a 60-90 min intravenous infusion every 3 weeks. In the combination regimen, irinotecan $\left(180-210 \mathrm{mg} / \mathrm{m}^{2}\right)$ was administered as $60-90 \mathrm{~min}$ intravenous infusion and 5 -FU $\left(500 \mathrm{mg} / \mathrm{m}^{2}\right.$, i.v. bolus $)$ modulated with folinic acid (FA) $\left(60 \mathrm{mg} / \mathrm{m}^{2}\right.$, i.v. bolus $)$. The $5-\mathrm{FU} / \mathrm{FA}$ administrations were repeated again the following day. The cycle was repeated every two weeks (8). The mean duration of chemotherapy was 6.5 months $( \pm 3.6)$. Treatment was continued until disease progression, or occurrence of unacceptable toxicity.

Fifteen out of $73(20.5 \%)$ tumours were located in the rectum. None of them have received neo-adjuvant radiotherapy since this was not the practice of our clinic at the time the patients were enrolled in the study.

The patients were prospectively followed-up until the end of March 2007; mean follow-up time from diagnosis was 32.6 months ( \pm 24.8 months). We used three endpoints to calculate the patient survival: a) disease-free survival (DFS); b) overall disease-specific survival (DSS) and c) survival with metastases (WMS). DFS is the time from diagnosis to the appearance of metastatic disease and is relevant only for those patients with radically operated stage II or III disease $(n=26)$. DSS is the time from diagnosis to death or to the time point when last seen alive, calculable for all patients in the study. WMS was calculated from the date of recording the disease recurrence/metastases at the clinical visit, until death or to the time point when last seen alive.

The study was approved by the TUH Ethics Committee and was conducted in accordance with the Declaration of Helsinki. Samples were collected with the endorsement of the National Authority for Medico-legal Affairs.

Immunohistochemical detection of Bcl-2 and hMLH1 and $h M S H 2$. Formalin-fixed, paraffin-embedded samples of the primary tumours were obtained from all 73 patients. Sections were cut serially at $5 \mu \mathrm{m}$ for routine haematoxylin and eosin staining and for immunohistochemical analysis. An experienced pathologist (K.S.) confirmed all histological diagnoses.

Bcl-2 protein expression was studied using monoclonal mouse anti-Bcl-2 antibody, which recognises a peptide comprising amino acids 41-54 of the human $\mathrm{Bcl}-2$ protein (Clone 124, Dako A/S, Glostrup, Denmark) (1:50). Expression
Table I. Characteristics of the patients and their tumours at diagnosis.

Variable Total patient group N (\%)

$\begin{array}{cr}\text { Patients } & 73(100) \\ \text { Female } & 27(37) \\ & 46(63)\end{array}$

Age (yrs)

Mean (SD)

$57.9(10.0)$

Tumour localisation

Rectum

Left colon

$31(42.5)$

Right colon

$20(27.4)$

Transverse colon

$7(9.6)$

Primary tumour status ${ }^{\mathrm{a}}$

T1

$\mathrm{T} 2$

T3

$46(63.0)$

T4

15 (20.5)

Primary nodal status ${ }^{\mathrm{a}}$

NO

20 (27.4)

N1

39 (53.4)

No data

Histological tumour grade

Grade I

Grade II

Grade III

Stage

II

III

Localisation of metastases

Liver

Lung

Multiple

Local

Disease-specific survival (months)

Mean (SD)

Disease-specific outcome

Alive

Died of disease

$62(84.9)$

${ }^{\mathrm{a}} \mathrm{TNM}$ classification (20).

of hMLH1 and hMSH2 was studied using antibodies against hMLH1 and hMSH2 (Mouse anti-MLH1, Clone 14 and Mouse anti-MSH2, Clone FE11, Zymed Laboratories Inc.) diluted in $1 \%$ bovine serum albumin/Tris-buffered saline 
(1:50 or 1:100, respectively). In brief, formalin-fixed paraffinembedded sections were de-paraffinised in xylene, rehydrated in graded alcohol, immersed in $0.01 \mathrm{M}$ citrate buffer ( $\mathrm{pH}$ 6.0), heated in a domestic microwave oven at full power for $2 \times 5 \mathrm{~min}$ and left in the buffer to cool to room temperature. The sections were incubated in $0.3 \%$ hydrogen peroxide for 20 min to block endogenous peroxidase activity. Incubations with the primary antibodies diluted in $1 \%$ bovine serum albumin/Tris-buffered saline, were carried out overnight in a humid chamber at $4^{\circ} \mathrm{C}$. The following day the slides were washed and incubated first with the biotinylated secondary antibody $\left(30 \mathrm{~min}, 20^{\circ} \mathrm{C}\right)$, then with avidin-biotin-peroxidase complex $\left(30 \mathrm{~min}, 20^{\circ} \mathrm{C}\right)$ (Vectastain ABC kit). Positive staining was visualised with 3,3' diaminobenzidine (DAB) substrate solution and the sections were counterstained with Mayer's haematoxylin. As negative controls, slides were processed with the omission of the primary antibodies.

Evaluation of Bcl-2 and hMLH1 and hMSH2 expression. Two observers (R.B., H.L.) assessed the expression of Bcl-2 and (hMLH1 and hMSH2) independently. Bcl-2 expression was first screened with low power for an overview of the general staining pattern. Four pictures of each slide covering most of the tumour area, were taken with a light microscope ( $\mathrm{x} 4$ magnification) connected to a camera and AnalySIS v 3.00 software (Soft Imaging System GmbH, Münster, Germany). Bcl-2 expression in the four pictures was analysed using (Imaging Research Inc., St. Catharine's, Ontario, Canada), which detected the brown colour of the positively stained tumour cells and counted the area of those cells (in pixels) and also counted the total tumour area. The percentage of the positively stained tumour cells from the whole tumour area was counted and used in further analysis. This method was able to distinguish between i) the presence of many cells expressing low amounts and ii) a few cells expressing high amounts of $\mathrm{Bcl}-2$, the $\mathrm{Bcl}-2$ expression reflecting total $\mathrm{Bcl}-2$ expression in the tumour, which is biologically more relevant.

Similarly, the expression of hMLH1 and hMSH2 was first screened to get an overview of the general staining pattern. Loss of expression was recorded when nuclear staining was absent from cancer cell nuclei but preserved in normal epithelium and stroma. Tumour expression was classified as weak, moderate or strong relative to the expression of normal epithelial and stromal cells present in each tumour section. Evaluation and scoring of the IHC slides was carried out without knowledge of the clinical data.

For statistical purposes, the expression profiles of each marker were treated as dichotomous variables, where tumours with negative or weak expression of either hMLH1 or hMSH2 were graded as one category (reduced expression) and all those with moderate or strong expression were included in the second category (normal expression). For Bcl-2, we used median values as cut-off to build up the dichotomous variable of low and high $\mathrm{Bcl}-2$ expression. In addition, combined Bcl-2/ MMR indices were created, using the dichotomous MMR variables and Bcl-2 variables (median cut-offs), resulting in four possible combinations of Bcl-2/MMR: low/low; low/high; high/low; and high/high. Then, these were converted to a 3-class index as follows: class $1, \mathrm{Bcl}-2 / \mathrm{MMR}$, low/low; class 2, Bcl-2/MMR, low/high or high/low; and class 3, Bcl-2/ MMR high/high.

Statistical analysis. Statistical analyses were performed using the SPSS ${ }^{\circledR}$ (SPSS, Inc., Chicago, IL, USA) and STATA (Stata Corp., TX, USA) software packages (SPSS for Windows, version 14.0.1 and STATA/SE 10.0). Frequency tables were analysed using the Chi-square test, with likelihood ratio (LR) or Fisher's exact test to assess the significance of the correlation between categorical variables. Differences in the means of continuous variables were analysed using nonparametric tests (Mann-Whitney or Kruskal-Wallis tests) for two- and multiple independent samples, respectively. ANOVA (analysis of variance) was only used for deriving the mean values $(95 \% \mathrm{CI})$ of each strata. Univariate survival (life-table) analysis for the outcome measure (DSS, DFS and WMS) was based on the Kaplan-Meier method and the groups were compared with the log-rank (Mantel-Cox) test. In all tests, the values $\mathrm{p}<0.05$ were regarded statistically significant.

\section{Results}

Bcl-2 and MMR (hMLH1 and hMSH2) expression levels were assessed using the median cut-off values and the lesions were classified as low and high expression categories (Fig. 1). In a bivariate correlation test, high $\mathrm{Bcl}-2$ expression was significantly related with both hMLH1 and MSH2 (Spearman test, $\mathrm{R}=0.367$, $\mathrm{p}=0.01$ ), when dichotomized as negative-weak (low) and moderate-strong (high). When analysed pair-wise, however, Bcl-2 expression and MLH1/MSH2 were significantly different (Wilcoxon signed ranks exact test; $\mathrm{p}=0.0001$ ).

In the next step, the different combined Bcl-2/MMR indices were related to all clinical variables recorded, including the response to treatment and patient survival. Bcl-2/MMR expression was significantly related to treatment response, as shown in Table II. Using the 3-class indices, tumours with low levels of both Bcl-2 and MMR responded better (58\%), as compared with those showing high Bcl-2 and MMR (13\%) $(\mathrm{p}=0.030)$. This same difference was even more accentuated among the 53 patients who received a combination of 5-FU/ FA with irinotecan; 68 and 18\%, respectively (Fisher's exact test, $\mathrm{p}=0.018$ ) (Table II).

A subset of patients (stage II, III) with high/high Bcl-2/ MMR expression presented with a significantly longer DFS than those with low/low Bcl-2/MMR expression (47 vs. 11 months) ( $\mathrm{p}=0.005$; ANOVA with LSD post-hoc test). In a univariate (Kaplan-Meier) survival analysis, this difference in DFS was also significant $(n=26 ; p=0.028)$ (Fig. 2). However, $\mathrm{Bcl}-2 / \mathrm{MMR}$ index was not significantly related to DSS or WMS in a univariate analysis (data not shown in figures).

Bcl-2 and MMR expression was not significantly associated with any other clinicopathological variables, including patient's age, gender, TNM status, grade, stage or carcinoembryonic antigen (CEA) levels.

\section{Discussion}

The treatment of colorectal cancer has become increasingly complex over recent years. With the emergence of new 
Table II. Bcl-2/MMR expression ${ }^{\mathrm{a}}$ and response to treatment in the whole series and in patients treated with combined (5-FU) plus irinotecan.

\begin{tabular}{lcc}
\hline All patients & No. of patients & Response N $(\%)$ \\
\hline Bcl-2/MMR index & & $18(58)$ \\
Both low & 31 & $12(46)$ \\
High/low or low/high & 26 & $3(18)$ \\
Both high & 16 & $33(45)$ \\
Total & 73 & Chi-square test (p=0.030)
\end{tabular}

Patients on combined 5-FU plus irinotecan

\section{Bcl-2/MMR index}

Both low

High/low or low/high

Both high

Total
25

17

11

53
$17(68)$

$11(65)$

$2(18)$

$30(56)$

Fisher's exact test $(\mathrm{p}=0.018)$

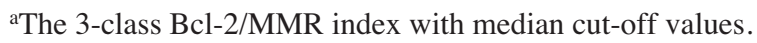
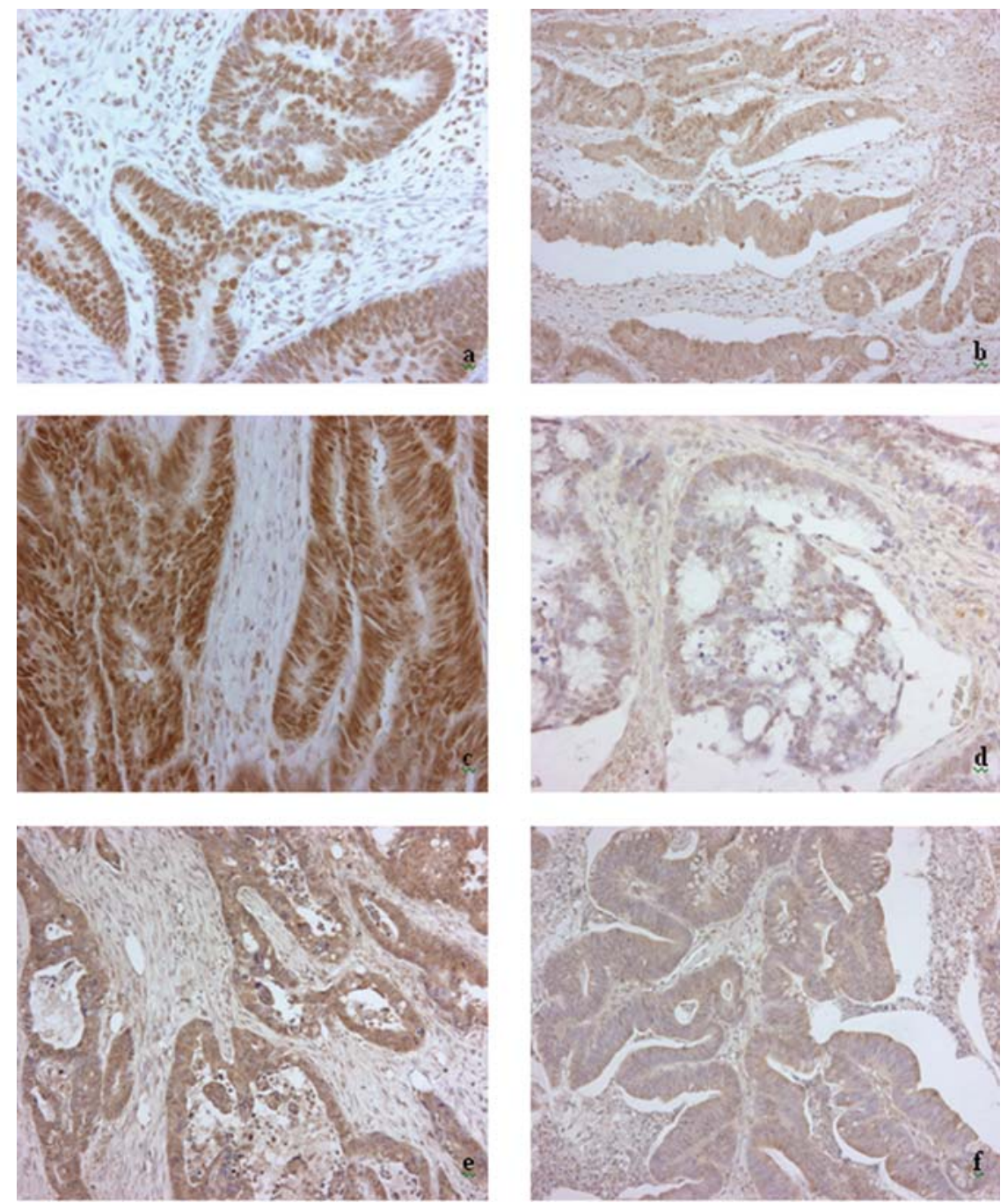

Figure 1. Examples of high (a), low (b) expression of hMLH1, and high (c), low (d) expression of hMSH2 (c and d). High/low staining and Bcl-2 staining (e and f) high/low (x20). 


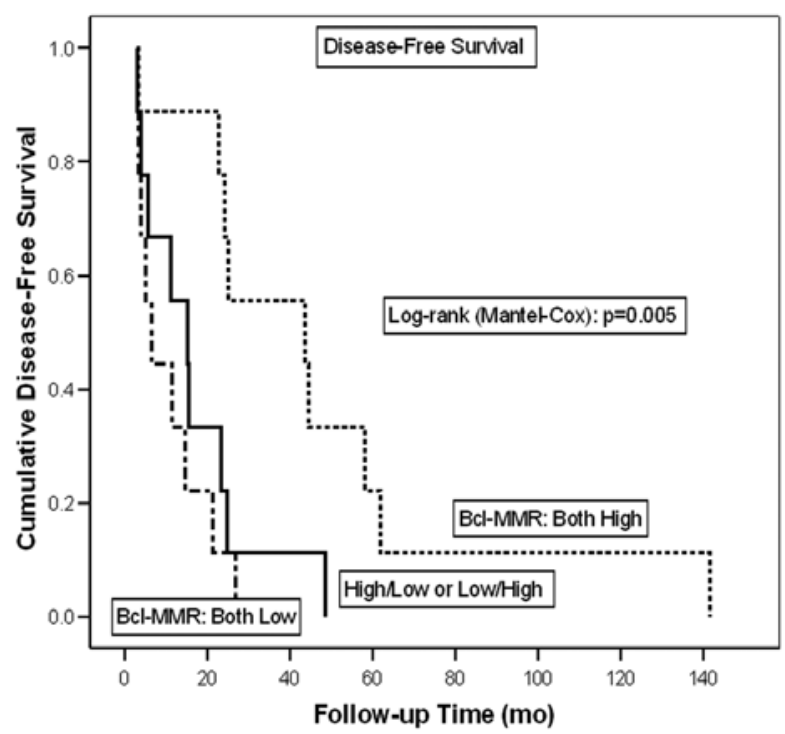

Figure 2. Bcl-2/MMR expression and disease-free survival (DFS) in univariate (Kaplan-Meier) analysis.

chemotherapy drugs and targeted agents, there has been a major improvement in the prognosis of patients with metastatic CRC. The identification of prognostic and predictive markers is clinically important, because CRC is a heterogeneous disease with various biological and clinical characteristics.

The present study analysed the combined Bcl-2 expression and MLH1 and MSH2 status in CRC, with particular reference to treatment response and disease outlook. The role of the Bcl-2 family of proteins in relation to chemoresponse has been evaluated extensively in vitro models. Overexpression of Bcl-2 and Bcl-xL has been shown to induce drug resistance (9-11). Furthermore, in relation to treatment, it has been demonstrated that elevated levels of the $\mathrm{Bcl}-2$ protein confers cytotoxic drug resistance to tumour cell lines $(4,12)$. Since Bcl-2 blocks apoptosis in vitro and thus contributes to malignant cell accumulation, its overexpression is expected to be associated with more aggressive tumour biology. Indeed, genetic alteration of the $\mathrm{Bcl}-2$ gene located on chromosome 18 is considered to be a key process in the pathogenesis and chemoresistence of human tumours, such as follicular lymphoma (13).

Assessing the DNA MMR status in CRC is relevant for several reasons: loss of one of the DNA MMR genes leads to rapid accumulation of mutations. In addition, an increased risk of tumorigenesis of colorectal cell lines deficient in MMR is associated with higher accumulation of other mutations and deletions $(7,14)$. Germ-line mutations in the MMR genes are known to result in hereditary non-polyposis colorectal cancer (HNPCC) and somatic alterations (15) or hypermethylation $(16,17)$ in this group of genes can result in the MSI found in $\sim 10-15 \%$ of CRCs $(18,19)$. Importantly, our study shows, for the first time the relationship between these two key molecules in a group of patients with locally advanced or metastatic CRC receiving similar treatment.

Of interest is the observed direct relationship between Bcl-2 and hMLH1, MSH2, with low vs. high levels of Bcl-2 correlating to those of hMLH1, MSH2 (Spearman R=0.367, $\mathrm{p}=0.01)$. This is the first study to establish such a direct relationship between MMR and Bcl-2. In pair-wise analysis, Bcl-2 expression and MLH1/MSH2 were significantly (Wilcoxon signed ranks test; $\mathrm{p}=0.0001$ ) different, however, implicating that in individual tumours, $\mathrm{Bcl}-2$ expression is not invariably associated with parallel changes in MMR, although these two issues are closely correlated in the whole series.

Our present results showed that tumours with low expression of both Bcl-2 and MMR (i.e. $\mathrm{MSI}^{+}$) appeared to be more sensitive to chemotherapy (Table II). Accordingly, of the 31 patients with tumours showing low Bcl-2 and MMR expression, $18(58 \%)$ demonstrated an objective treatment response, as compared with only $3(18 \%)$ of those with high Bcl-2-MMR expression. This difference was even more marked in the group of patients $(n=53)$ who received 5-FU containing therapy; 68 vs. $18 \%(\mathrm{p}=0.018)$ demonstrated an objective treatment response. In light of these data, it seems feasible to speculate that tumour cells' ability to repair DNA damage (i.e., their MMR status) influences their response to chemotherapeutic agents that cause DNA damage. One would expect that, if this capacity is well retained (i.e., high MMR index), the response to DNA damaging agents (such as 5-FU) would be more modest than in cases where MMR fails (i.e., low MMR index). The latter should lead to accumulation of mutations that might increase the sensitivity of the tumour cells to cytotoxic agents. However, there may also be reduced sensitivity to treatment although DNA repair mechanisms are functioning, if the normal apoptotic response to accumulated DNA damage is dysregulated.

As to the patient survival, there was a significant correlation of Bcl-2/MMR expression with DFS, in that the patients with high Bcl-2/MMR index had a longer DFS (47 vs. 11 months, respectively) (Fig. 2). No such effect was shown in DSS, which was not significantly different among the patients with low and high Bcl-2/MMR indices. Similarly, these high indices did not show any significant benefit in the survival time with metastases (WMS). To interpret these findings one must remember that a high $\mathrm{Bcl}-2 /$ MMR index means that MMR protein expression is present (i.e. normal) but that $\mathrm{Bcl}$ expression is high (abnormal). This suggests that the beneficial effects of high Bcl-2/MMR index are only relevant to disease before metastasis has occurred.

The practical implications of our observations are two-fold; patients with high $\mathrm{Bcl}-2 / \mathrm{MMR}$ expression seem to have a significantly longer DFS than those with low Bcl-2/MMR index. On the other hand, tumours with high expression seem to respond poorly to the therapeutic regimes used. Then, patients with low Bcl-2/MMR index are likely to be good responders to chemotherapy.

To conclude, the present data suggest that patients with advanced and metastatic CRC whose tumours have low Bcl-2 and deficient MMR demonstrate a significantly shorter DFS, as compared to patients with high expression of these markers. However, these patients with low Bcl-2 and MMR deficient tumours are also more likely to obtain the greatest benefit from chemotherapy. 


\section{References}

1. Boyle P and Ferlay J: Cancer incidence and mortality in Europe, 2004. Ann Oncol 16: 481-488, 2005.

2. Bronner MP, Culin C, Reed JC and Furth EE: The bcl-2 protooncogene and the gastrointestinal epithelial tumor progression model. Am J Pathol 146: 20-26, 1995.

3. Sinicrope FA, Ruan SB, Cleary KR, Stephens LC, Lee JJ and Levin B: bcl-2 and p53 oncoprotein expression during colorectal tumorigenesis. Cancer Res 55: 237-241, 1995.

4. Hockenbery D, Nunez G, Milliman C, Schreiber RD and Korsmeyer SJ: Bcl-2 is an inner mitochondrial membrane protein that blocks programmed cell death. Nature 348: 334-336, 1990.

5. Pidgeon GP, Barr MP, Harmey JH, Foley DA and Bouchier-Hayes DJ: Vascular endothelial growth factor (VEGF) upregulates BCL-2 and inhibits apoptosis in human and murine mammary adenocarcinoma cells. $\mathrm{Br} \mathrm{J}$ Cancer 85 : 273-278, 2005.

6. Kawasaki H, Toyoda M, Shinohara H, Okuda J, Watanabe I, Yamamoto T, Tanaka K, Tenjo T and Tanigawa N: Expression of survivin correlates with apoptosis, proliferation, and angiogenesis during human colorectal tumorigenesis. Cancer 91: 2026-2032, 2001

7. Haydon AM and Jass JR: Emerging pathways in colorectalcancer development. Lancet Oncol 3: 83-88, 2002.

8. Glimelius B, Ristamaki R, Kjaer M, Pfeiffer P, Toren JS, Tveit KM, Linne T, Frodin JE, Boussard B, Oulid-Aissa D and Pyrhonen S: Irinotecan combined with bolus 5-fluorouracil and folinic acid Nordic schedule as first line therapy in advanced colorectal cancer. Ann Oncol 13: 1868-1873, 2002.

9. Miyashita T and Reed JC: bcl-2 gene transfer increases relative resistance of S49.1 and WEHI7.2 lymphoid cells to cell death and DNA fragmentation induced by glucocorticoids and multiple chemotherapeutic drugs. Cancer Res 52: 5407-5411, 1992.

10. Minn AJ, Rudin CM, Boise LH and Thompson CB: Expression of bcl-xL can confer a multidrug resistance phenotype. Blood 86: 1903-1910, 1995
11. Walton MI, Whysong D, O'Connor PM, Hockenbery D, Korsmeyer SJ and Kohn KW: Constitutive expression of human Bcl-2 modulates nitrogen mustard and camptothecin induced apoptosis. Cancer Res 53: 1853-1861, 1993.

12. Leahy DT, Mulcahy HE, O'Donoghue DP and Parfrey NA: bcl-2 protein expression is associated with better prognosis in colorectal cancer. Histopathology 35: 360-367, 1999.

13. McDonnell TJ and Korsmeyer SJ: Progression from lymphoid hyperplasia to high-grade malignant lymphoma in mice transgenic for the $\mathrm{t}(14 ; 18)$. Nature 349: 254-256, 1991.

14. Mori Y, Yin J, Rashid A, Leggett BA, Young J, Simms L, Kuehl PM, Langenberg P, Meltzer SJ and Stine OC: Instabilotyping: comprehensive identification of frameshift mutations caused by coding region microsatellite instability. Cancer Res 61: 6046-6049, 2001.

15. Thibodeau SN, French AJ, Cunningham JM, Tester D, Burgart LJ, Roche PC, McDonnell SK, Schaid DJ, Vockley CW, Michels VV, Farr GH Jr and $\mathrm{O}^{\prime}$ Connell MJ: Microsatellite instability in colorectal cancer: different mutator phenotypes and the principal involvement of hMLH1. Cancer Res 58: 1713-1718, 1998.

16. Cunningham JM, Christensen ER, Tester DJ, Kim CY, Roche PC, Burgart LJ and Thibodeau SN: Hypermethylation of the hMLH1 promoter in colon cancer with microsatellite instability. Cancer Res 58: 3455-3460, 1998

17. Kane MF, Loda M, Gaida GM, Lipman J, Mishra R, Goldman H, Jessup JM and Kolodner R: Methylation of the hMLH1 promoter correlates with lack of expression of hMLH1 in sporadic colon tumors and mismatch repair-defective human tumor cell lines. Cancer Res 57: 808-811, 1997.

18. Lengauer C, Kinzler KW and Vogelstein B: Genetic instabilities in human cancers. Nature 396: 643-649, 1998.

19. Halford S, Sasieni P, Rowan A, Wasan H, Bodmer W, Talbot I, Hawkins N, Ward R and Tomlinson I: Low-level microsatellite instability occurs in most colorectal cancers and is a nonrandomly distributed quantitative trait. Cancer Res 62: 53-57, 2002.

20. Parkin DM: Global cancer statistics in the year 2000. Lancet Oncol 2: 533-543, 2001. 